\title{
Elementary example of exact effective-Hamiltonian computation
}

\author{
Stanisław D. Głazek®* \\ Institute of Theoretical Physics, Faculty of Physics, University of Warsaw, \\ Pasteura 5, 02-093 Warsaw, Poland
}

(Received 30 October 2020; accepted 4 January 2021; published 21 January 2021)

\begin{abstract}
We present an exact computation of effective Hamiltonians for an elementary model obtained from the Yukawa theory by going to the limit of bare fermions being infinitely heavy and bare bosons being at rest with respect to the fermions that emit or absorb them. The coupling constant can be arbitrarily large. The Hamiltonians are computed by solving the differential equation of the renormalization group procedure for effective particles (RGPEP). Physical fermions, defined in the model as eigenstates of the effective Hamiltonians, are obtained in the form of an effective fermion dressed with a coherent state of effective bosons. The model computation illustrates the method that can be used in perturbative computations of effective Hamiltonians for realistic theories. It shows the mechanism by which the perturbative expansion and Tamm-Dancoff approximation increase in accuracy along the RGPEP evolution.
\end{abstract}

DOI: 10.1103/PhysRevD.103.014021

\section{INTRODUCTION}

Complexity of relativistic quantum field theory (QFT) implies a need for approximate computational methods. One needs a systematic scheme for improving their accuracy. That is the case in computing observables using expansion in powers of a small coupling constant, solving eigenvalue problems using a limited basis in the space of states or using renormalization group methods. A combination of all three of these techniques requires a clear-cut pattern to follow. Such pattern can only be provided by an exactly solvable model, because one needs the exact solution to unambiguously assess accuracy of the approximate calculations. On the other hand, to obtain an exactly solvable model, one has to simplify a theory. A compromise needs to be struck between simplifying and obtaining a helpful pattern.

This paper presents a novel, exact renormalization group computation of effective Hamiltonians in a model that results from drastic but precisely specified simplifications of QFT, so that one can see the steps that would have to be reconstructed in an analogous computation of the effective Hamiltonians and their spectra in QFT. The presentation is thus quite limited but it includes enough of the QFT features for addressing the issues of high orders of perturbation theory, few-body approximations in the

\footnotetext{
*stglazek@fuw.edu.pl
}

Published by the American Physical Society under the terms of the Creative Commons Attribution 4.0 International license. Further distribution of this work must maintain attribution to the author(s) and the published article's title, journal citation, and DOI. Funded by SCOAP ${ }^{3}$.
Fock space, renormalization-group improvements, and the form of effective Hamiltonians that change, but not limit the number of interacting field quanta.

The computation presented in this paper concerns a model that is obtained by drastically simplifying the Yukawa theory. The simplifications made here partly resemble the ones that Wilson adopted in formulating his approach to renormalization using a Yukawa-like model [1], but they go much further. As a result, the ultraviolet divergences of a local theory are eliminated at the outset. This is useful because the goal of the presented computation is not to find the ultraviolet counterterms using the triangle of renormalization [2], as it was in Wilson's case, but to deal with the issue of computation of an effective theory Hamiltonian after the right counterterms have already removed the divergences. The model computation includes a pattern of handling terms that are analogous to the finite parts of counterterms.

It should be stressed that the history of models that incorporate elements of the Yukawa theory and are helpful in understanding renormalization in QFT beyond the weakcoupling expansion have a long history [3]. There are exactly solvable models among them, e.g., [4]. Also, a model may employ some elements of the Yukawa theory formalism and be exactly solvable without encountering any need for renormalization. For example, a class of twolevel models for a system of a fixed number of fermions, whose Hamiltonians can be written using bilinear products of fermion creation and annihilation operators, could be solved exactly. One takes advantage of the SU(2) symmetry associated with the two levels $[5,6]$ or uses the symmetry of the model's boson representation [7]. One can even show that such fermion systems exhibit thermalization when they 
are weakly coupled to a boson bath [8]. This variety of models that can be solved suggests to the author it should be clearly stated that the main purpose here is different. It is to apply a recently formulated renormalization group equation for Hamiltonians of QFT, to a simplified, onelevel model for fermions coupled to bosons in a way that is analogous to the Yukawa theory coupling. In the model case, an exact operator solution to the equation is obtained in the form of a whole family of effective Hamiltonians that are strictly equivalent. They all act in an infinite dimensional Fock space. Their common spectrum is obtained as a byproduct of the solution to the renormalization group equation. Comparisons and comments concerning the most similar models known to the author are provided in Sec. VIII.

The model used here is defined using the front form (FF) of Hamiltonian dynamics [9] instead of the instant form (IF) used in [1]. It is known that to obtain the Wilson model from the front form of Yukawa theory one needs to consider the limit of fermions that are much heavier than the momentum cutoff parameter [10], say $\Lambda$. Here, in addition, also the boson mass is assumed much greater than $\Lambda$. This limit is called the static limit, since bosons emitted or absorbed by fermions do not move with respect to their source. Further, the model we use does not include isospin, which leads to a significant simplification: only four distinct operators appear in the effective Hamiltonians. This feature will become clear in the course of computation. Despite these far reaching simplifications, the model interaction Hamiltonian changes the number of bosons and the number of Fock components involved in the dynamics is infinite.

To compute the effective Hamiltonians, we use the method called the renormalization group procedure for effective particles (RGPEP). The RGPEP differs conceptually from the Wilson renormalization group procedure. Namely, instead of integrating out high-energy modes in the basis of the space of states in which the Hamiltonian acts, one changes the basis in the space of operators to which the Hamiltonian belongs. In other words, the Hamiltonian is seen as an element of the operator space formed by normal-ordered polynomials of bare creation and annihilation operators. The change of basis in the space of such polynomials is obtained by replacing the bare creation and annihilation operators with the analogous ones for the effective quanta of fields, called effective particles; see Sec. III for details. In perturbation theory, the effective particle operators are polynomials in terms of the bare particle operators and vice versa [11]. The interactions of effective particles are limited by the running cutoff $\Lambda$ that provides an upper bound on the magnitude of the invariant mass change that an interaction can cause. The RGPEP evolution of the computed Hamiltonians describes the variation of their form with the running cutoff.

The RGPEP employs the rules of the similarity renormalization group procedure for Hamiltonians $[12,13]$ and takes advantage of the double-commutator feature of Wegner's flow equation for Hamiltonian matrices [14]. In application to local QFT, the RGPEP has been recently illustrated in [15], which also includes references to the previous works. However, in all these examples, one is forced to use the approximations that are not under precise control, such as the mentioned earlier weak-coupling expansion [2] or a limitation on a number of virtual particles, called the Tamm-Dancoff (TD) approximation $[16,17]$. These approximations obscure the core features of the RGPEP in the context of realistic theories. In contrast, the exact RGPEP computation of effective Hamiltonians in the model described here is quite transparent and the result has a clear interpretation in terms of the Fock-space image of physical states.

The RGPEP equation we solve, see Eq. (15), determines the evolution of Hamiltonians using not the cutoff parameter $\Lambda$ itself, but the parameter that is denoted by $t$ and corresponds to $\Lambda^{-2}$. Thus, $t$ varies from zero for the initial Hamiltonian to infinity for its final, diagonal form, in which all mass-changing interaction terms disappear. The ability to diagonalize Hamiltonians is the key design feature of the RGPEP equation. Quite generally, the design secures that the first-order solution of the RGPEP evolution equation results in vertex form factors whose width in momentum variables varies with $t$. The width tends to infinity or some cutoff value when $t \rightarrow 0$ and to zero when $t \rightarrow \infty$. In QFT, these form factors regulate singularities of the local interactions, e.g., see [15], and can be thought of as corresponding to a finite size of the effective particles. However, in the model solved here, the situation is much simpler because of the static limit. One only obtains a running-coupling constant, denoted by $g_{t}$, instead of a function of momentum, since the interacting particles are at rest with respect to each other.

The paper is organized in the following way. Section II describes the model Hamiltonian, cf. [3]. The model is derived in the FF of dynamics using the static limit of the Yukawa theory in Sec. II A. It is rewritten in a more familiar energy notation of the IF of dynamics in Sec. II B. Section III describes solution of the RGPEP equations. First, the equations design is explained in Sec. III A and then the discussion of solutions follows in Sec. III B. Operators that create and annihilate effective particles are derived in Sec. IV, with final formulas in Sec. IVA. The exact spectrum of the model Hamiltonian in the Fockspace representation is given in Sec. V. The issue of approximate computations is addressed in the remaining part of the paper. Section VI discusses the weak-coupling expansion. The TD approximation is discussed in Sec. VII. Subsequently, Sec. VIII introduces the concept of effective TD Hamiltonian matrices, including comments and comparisons to related work on similar models. Section IX briefly outlines the ways of comparing the model solution with realistic theories. Section X concludes the paper and reviews motivation for studies of QFT using the RGPEP. 


\section{MODEL HAMILTONIAN}

The model Hamiltonian we consider is obtained from the Yukawa theory using results of Ref. [10]. In that work, Eq. (2.1) displays the canonical front-form Hamiltonian of Yukawa theory that has the structure

$$
H_{c}=H_{f}+H_{b}+H_{f b}+[\text { other terms }],
$$

where $H_{f}$ stands for the Hamiltonian of free fermions of mass $m$,

$$
H_{f}=\sum_{\sigma} \int[p] \frac{m^{2}+p^{\perp 2}}{p^{+}} b_{p \sigma}^{\dagger} b_{p \sigma}
$$

$H_{b}$ denotes a free Hamiltonian for bosons of mass $\mu$,

$$
H_{b}=\sum_{\sigma} \int[p] \frac{\mu^{2}+p^{\perp 2}}{p^{+}} a_{p}^{\dagger} a_{p},
$$

$H_{f b}$ is the fermion-boson interaction term,

$$
\begin{aligned}
H_{f b}= & g \sum_{\sigma_{1}, \sigma_{2}} \int\left[p_{1} p_{2} p_{3}\right] \delta_{c . a} \bar{u}_{1} \Gamma u_{2} \\
& \times b_{p_{1} \sigma_{1}}^{\dagger}\left(a_{p_{3}}^{\dagger}+a_{p_{3}}\right) b_{p_{2} \sigma_{2}} .
\end{aligned}
$$

The bracket [other terms] indicates the terms that disappear in comparison with the first three in the limit of the fermion mass $m \rightarrow \infty$. The symbol $[p]$ conventionally denotes the measure $d^{2} p^{\perp} d p^{+} \theta\left(p^{+}\right) /\left[2 p^{+}(2 \pi)^{3}\right]$ and $\delta_{a . c}$ is $2(2 \pi)^{3} \delta^{3}\left(P_{c}-P_{a}\right)$, where $P_{c}$ and $P_{a}$ denote the total + and $\perp$ momenta of the bare particles that are created and annihilated by the interaction, respectively. Fermion spinors are denoted by $u_{1}$ and $u_{2}$ and the matrix $\Gamma$ is set to 1 . The coupling constant is denoted by $g$. Further model construction steps use only the first three terms in Eq. (1).

\section{A. The static limit}

As a result of steps fully described in [10], the Hamiltonian $H=H_{f}+H_{b}+H_{f b}$ is altered in a way that leads to a formula resembling Eq. (2.17) in that reference. One considers a fermion eigenstate of $H$ that carries an arbitrary momentum $P^{+}$and $P^{\perp}$ and a fixed value of spin projection on $z$-axis. The state is a combination of the Fock component with one bare fermion in the same spin state and infinitely many Fock components each of which contains one bare fermion and some natural number of bare bosons. The fermion mass eigenvalue is written as $M=m+E$, where $E / m \ll 1$. Every boson kinematic momentum in a Fock component with $n$ bosons is parametrized according to the rule

$$
p_{n, i}^{+}=y_{n, i} P^{+},
$$

$$
p_{n, i}^{\perp}=y_{n, i} P^{\perp}+\kappa_{n, i}^{\perp},
$$

where $0<i \leq n$. The corresponding fermion momentum is given by

$$
\begin{gathered}
p_{n}^{+}=x_{n} P^{+}, \\
p_{n}^{\perp}=x_{n} P^{\perp}-\kappa_{n, i}^{\perp} \ldots-\kappa_{n, i}^{\perp},
\end{gathered}
$$

where

$$
x_{n}=1-y_{n, 1} \ldots-y_{n, n} .
$$

In the absence of bosons, the bare fermion carries the whole $P^{+}$and $P^{\perp}$. Approximations described in [10] are based on the conditions that force $\kappa_{n, i}^{\perp} \ll m$ and $\sum_{i=1}^{n} y_{n, i} \ll 1$. A sufficient condition is provided by imposing a cutoff that forces all bosons to only have momenta relative to the fermion that are negligible in comparison with the fermion mass.

The interaction Hamiltonian $H_{f b}$ is supplied with a cutoff form factor, denoted below by $f_{\Lambda}$. One assumes that $\Lambda \ll m$. The cutoff function enforces the condition $\left|P_{c}^{2}-P_{a}^{2}\right|<\Lambda^{2} \ll m^{2}$, where $P_{c}$ and $P_{a}$ denote the free total four-momenta of created and annihilated particles, respectively. In consequence, all the fractions $y_{i}$ defined above tend to 0 and the fermion fractions $x_{n} \rightarrow 1$. The resulting Hamiltonian that determines the mass eigenvalue $M$ for a physical fermion, see Eqs. (2.16) and (2.17) in [10], takes the form

$$
H_{\text {fermion }}=m b^{\dagger} b+H_{1} b^{\dagger} b,
$$

where $b$ denotes annihilation operator for a bare fermion at rest and only one spin projection on $z$-axis. The operator $H_{1}$ is the boson Hamiltonian associated with the states that contain one bare fermion,

$$
\begin{aligned}
H_{1}= & \int[q]\left[(1 / 2)\left(q^{+}+\frac{\mu^{2}+q^{\perp 2}}{q^{+}}\right) a_{q}^{\dagger} a_{q}\right. \\
& \left.+g f_{\Lambda}(q)\left(a_{q}^{\dagger}+a_{q}\right)\right] .
\end{aligned}
$$

The boson momenta in all Fock sectors are identified according to the same relations $q^{+}=y_{n, i} m$ and $q^{\perp}=\kappa_{n, i}^{\perp}$. The half of the round bracket in Eq. (11) equals energy of a boson with momentum $\vec{q}$ in which $2 q^{z}=q^{+}-$ $\left(\mu^{2}+q^{\perp 2}\right) / q^{+}$. It is possible to determine the allowed momenta for bosons, including the sampling that Wilson adopted, by choosing the function $f_{\Lambda}(q)$. At this point, one can further proceed as in [10] and show that when the initial Yukawa theory includes isospin, the Hamiltonian one obtains in place of $H_{1}$ also includes isospin and matches the model 
Hamiltonian studied in [1]. In what follows, a different path is taken.

The next great simplification step we make here, which was not made in [10], is to assume that the boson mass $\mu$ is also much larger than the cutoff $\Lambda$ on $|\vec{q}|$. This assumption implies that the bosons cannot move with respect to the fermion that emits or absorbs them. In the limit $\Lambda / \mu \rightarrow 0$, the Hamiltonian $H_{1}$ involves only bosons that are practically at rest relative to the fermion. One replaces all bosons nearly at rest with respect to the fermion by just one static boson mode, for which $q^{\perp}=0$ and $q^{+}=\mu$. We consider the model in which $\mu / m \rightarrow 0$. Finally, one can allow the static bosons to appear in the model also without a fermion. This way one arrives at the Hamiltonian of a model for which the exact effective Hamiltonians are computed using the RGPEP in the sections that follow.

\section{B. Intuitive notation}

The model Hamiltonian introduced in the previous section is rewritten here using an intuitive notation that does not require familiarity with the front form of dynamics and instead relies on the intuition rooted in the IF of dynamics,

$$
H=E_{f} b^{\dagger} b+E_{b} a^{\dagger} a+g E_{I} b^{\dagger}\left(a^{\dagger}+a\right) b,
$$

where $E_{f}, E_{b}$, and $E_{I}$ are the fermion, boson, and interaction energy parameters, $g$ is a coupling constant, while $b$ and $a$ are annihilation operators for the static fermion and boson, respectively. These operators and their Hermitian conjugates are normalized to obey the standard (anti)commutation relations, of which the only nonzero ones are

$$
\begin{gathered}
\left\{b, b^{\dagger}\right\}=1, \\
{\left[a, a^{\dagger}\right]=1 .}
\end{gathered}
$$

The Hamiltonian describes fermions of just one spin state and preserves their number, which can only be 0 or 1 . The number of bosons is neither specified nor limited and it varies as a result of interactions.

In states with the fermion number equal zero, the interaction vanishes and the spectrum matches the one of a Hamiltonian for free bosons at rest, $H_{b}=E_{b} a^{\dagger} a$, with eigenvalues $E_{b n}=n E_{b}$, where $n$ is zero or a natural number. The corresponding normalized eigenstates of $H_{b}$ are $|n\rangle=(n !)^{-1 / 2} a^{\dagger n}|0\rangle$. In states with the fermion number equal 1, the Hamiltonian changes the boson number by 1 and the distribution of bosons needs to be computed.

We apply the RGPEP to this model in the remaining part of this work. This means that instead of directly evaluating all of the Hamiltonian eigenvalues and eigenstates in terms of bare quanta, one introduces creation and annihilation operators for effective bosons and fermions and computes the effective Hamiltonians for them. The eigenstates of these effective Hamiltonians are then found in terms of the basis in the Fock space that is constructed using the creation operators of the effective particles instead of the bare ones. The exercise is meant worth carrying out since one can unfold the simplifications used in deriving $H$ of Eq. (12) and look at the dynamics of Yukawa theory anew from the perspective of the model computation.

\section{COMPUTATION OF THE EFFECTIVE HAMILTONIAN}

In the model considered here, the RGPEP equations for a family of renormalized Hamiltonians, labeled by parameter $t$, can be written in the operator form,

$$
\begin{gathered}
\frac{d}{d t} \mathcal{H}_{t}=\left[\mathcal{G}_{t}, \mathcal{H}_{t}\right], \\
\mathcal{G}_{t}=\left[H_{f}+H_{b}, \mathcal{H}_{t}\right],
\end{gathered}
$$

where $\mathcal{G}_{t}$ is called the generator. The initial condition at $t=0$ is provided by $H$ of Eq. (12), which is denoted for that reason as $H_{0} . H_{f}$ equals $E_{f} b^{\dagger} b$.

Equations (15) and (16) resemble Wegner's flow equations that describe the evolution of band-diagonal Hamiltonian matrices as functions of their width on energy scale; the width decreases as $t$ increases [14]. There are two differences. One is that Eq. (15) cannot be represented exactly by finite matrices, because the commutation relations for $a$ and $a^{\dagger}$ cannot. The other one is that the generator $\mathcal{G}_{t}$ is a commutator of $\mathcal{H}_{t}$ with the sum $H_{f}+H_{b}$ that does not depend on $t$, cf. [18]. In the Wegner generator, the Hamiltonian matrix is commuted with its diagonal part that varies with $t$. It should be noted that Eq. (15) is written for the operator $\mathcal{H}_{t}$ that only contains $t$-independent creation and annihilation operators for bare particles, which are replaced by the corresponding $t$-dependent operators for effective particles in order to obtain the renormalized Hamiltonians $H_{t}$; see below.

\section{A. Design of Eqs. (15) and (16)}

Design of Eqs. (15) and (16) originates in the idea that one can consider the Hamiltonian eigenvalue problems in local QFT in terms of some kind of effective quanta instead of the bare ones. The change from bare to effective quanta is motivated by the concept that the effective quanta interact in a so much less violent way than the bare quanta do that the eigenvalue problem may be convergent in the effective Fock-space basis, even if it does not exhibit convergence in the bare Fock-space basis. The appearance of convergence is a consequence of the vertex factors that emerge in solutions of Eq. (15). Emergence of such factors is the feature of double-commutator equations like Eq. (15) with the generator given by Eq. (16). The model application 
discussed here shows this feature in a simplified way, see below, and demonstrates how convergence in the effective Fock-space basis improves with increase of $t$.

The key examples of physical elementary particle systems in terms of which one can think about the design of Eqs. (15) and (16) are hadrons. In QCD, represented in terms of bare quanta, hadrons are complex mixtures of infinitely many quarks and gluons that are confined. In the particle tables, most of the known hadrons are classified as bound states of just a few constituent quarks. The design of the RGPEP equations can be described as aiming at the derivation of a mathematically precise connection between these two pictures of hadrons.

The creation and annihilation operators for effective particles are defined using a unitary transformation of the form

$$
q_{t}=\mathcal{U}_{t} q \mathcal{U}_{t}^{\dagger},
$$

where $q$ stands for the operators $a, a^{\dagger}, b$, or $b^{\dagger}$, and

$$
\mathcal{U}_{t}^{\dagger}=T \exp \left(\int_{0}^{t} d \tau \mathcal{G}_{\tau}\right)
$$

The symbol $T$ denotes ordering in $\tau$. The Hamiltonian operator

$$
H_{t}=\mathcal{U}_{t} \mathcal{H}_{t} \mathcal{U}_{t}^{\dagger}
$$

is defined to be the same as the initial one, $H_{t}=H_{0}$, but $H_{t}$ is expressed in terms of the effective particle operators $a_{t}$, $a_{t}^{\dagger}, b_{t}$, and $b_{t}^{\dagger}$ instead of the initial operators $a, a^{\dagger}, b$, and $b^{\dagger}$ that correspond to $t=0$. Thus, in $H_{t}$, the coefficients of products of the effective creation and annihilation operators are different from the coefficients of products of the corresponding initial operators in $H_{0}$. The coefficients in $H_{t}$ contain factors that follow from the double-commutator structure of Eq. (15). These factors are obtained in the process of solving Eq. (15). They emerge in a way similar to the emergence of the band-diagonal matrices from the Wegner flow equation.

If the model were divergent, as it is the case for bare Hamiltonians in local QFT, $H_{0}$ would be supplied with the counterterms that would be computed from the condition that the coefficients of effective particle operators in $H_{t}$ for any finite, fixed value of $t$ are not sensitive to the adopted regularization of the divergences. Since the model Hamiltonian of Eq. (12) does not generate divergences, the computation of counterterms to divergent expressions is not needed and this aspect of local QFT is not illustrated in the model solution. The divergence counterterm computation in QFT significantly complicates the RGPEP procedure with a lot of details that depend on the adopted regularization. These largely arbitrary details obstruct the conceptual view of the method while the model computation makes it clear. Counterterms appear in the model computation only in a finite form, which is analogous to the appearance of the unknown finite parts of the divergence counterterms in local QFT.

\section{B. Solution of Eq. (15)}

In order to solve Eq. (15), one writes

$$
\begin{aligned}
\mathcal{H}_{t}= & \left(E_{f}+\delta E_{f t}\right) b^{\dagger} b+E_{b} a^{\dagger} a \\
& +g_{t} E_{I} b^{\dagger}\left(a^{\dagger}+a\right) b,
\end{aligned}
$$

where the subscript $t$ indicates dependence on that argument. Only four distinct Fock-space operators appear in this formula because no other operators are generated from the initial condition of Eq. (12). Using a dot to indicate the derivative, one obtains Eq. (15) in the form

$$
\begin{aligned}
\delta \dot{E}_{f t} b^{\dagger} b+\dot{g}_{t} E_{I} b^{\dagger}\left(a^{\dagger}+a\right) b & \\
= & {\left[\mathcal{G}_{t},\left(E_{f}+\delta E_{f t}\right) b^{\dagger} b+E_{b} a^{\dagger} a\right.} \\
+ & \left.g_{t} E_{I} b^{\dagger}\left(a^{\dagger}+a\right) b\right], \\
\mathcal{G}_{t} & =g_{t} E_{b} E_{I} b^{\dagger}\left(a^{\dagger}-a\right) b .
\end{aligned}
$$

The generator takes the simple form since the fermion number is conserved by the interaction. Evaluation of the commutator on the right-hand side of Eq. (21) yields

$$
\begin{gathered}
\delta \dot{E}_{f t} b^{\dagger} b+\dot{g}_{t} E_{I} b^{\dagger}\left(a^{\dagger}+a\right) b \\
=-g_{t} E_{b}^{2} E_{I} b^{\dagger}\left(a^{\dagger}+a\right) b \\
-2 g_{t}^{2} E_{b} E_{I}^{2} b^{\dagger} b .
\end{gathered}
$$

Equating coefficients in front of the same operators on both sides, one gets

$$
\begin{gathered}
\delta \dot{E}_{f t}=-2 g_{t}^{2} E_{b} E_{I}^{2}, \\
\dot{g}_{t}=-g_{t} E_{b}^{2} .
\end{gathered}
$$

These are ordinary differential equations and solving them leads to the solution of Eq. (15) in the form

$$
\begin{aligned}
\mathcal{H}_{t}= & {\left[E_{f}+g_{t}^{2} \Delta_{t}\right] b^{\dagger} b+E_{b} a^{\dagger} a } \\
& +g_{t} E_{I} b^{\dagger}\left(a^{\dagger}+a\right) b,
\end{aligned}
$$

where

$$
\begin{gathered}
g_{t}=g e^{-E_{b}^{2} t}, \\
\Delta_{t}=\left(1-e^{2 E_{b}^{2} t}\right) E_{I}^{2} / E_{b} .
\end{gathered}
$$

This result shows that the increase of $t$ from zero to infinity causes the effective fermion-boson coupling constant $g_{t}$ to 
decrease exponentially fast from its initial value $g$ to zero at the rate given by an inverse of the boson energy squared. This is the promised suppression of interactions by the vertex factor. One obtains the vertex factor in this model solely in the form of a varying coupling constant $g_{t}$ instead a whole form factor that is a function of momentum and energy transfer between quanta in the vertex. The simplification occurs because the model contains only static modes for fermions and bosons.

The boson energy $E_{b}$ stays constant as a function of $t$. The fermion energy, $E_{f}+g_{t}^{2} \Delta_{t}$, evolves from the initial value $E_{f}$ to the final fermion eigenvalue energy

$$
E_{f \infty}=\lim _{t \rightarrow \infty} E_{f}+g_{t}^{2} \Delta_{t}=E_{f}-g^{2} E_{I}^{2} / E_{b} .
$$

It seems that $E_{f \infty}$ may be negative. However, it could only happen outside the range of approximations made in the model, where the fermion energy $E_{f}$ is assumed much larger than the boson energy $E_{b}$ and much larger than the energy change due to the interaction, $g E_{I}$, while $E_{b}$ and $E_{I}$ are of similar magnitude. Therefore, for any fixed value of $g$, one only considers $E_{f}$ much larger than $g^{2} E_{I}^{2} / E_{b}$.

\section{EFFECTIVE PARTICLES}

Solution for the operator $\mathcal{H}_{t}$ in Eq. (26) is transformed into the Hamiltonian for effective particles using the operator $\mathcal{U}_{t}$ according to Eq. (19). The result is

$$
\begin{aligned}
H_{t}= & \left(E_{f}+g_{t}^{2} \Delta_{t}\right) b_{t}^{\dagger} b_{t}+E_{b} a_{t}^{\dagger} a_{t} \\
& +g_{t} E_{I} b_{t}^{\dagger}\left(a_{t}^{\dagger}+a_{t}\right) b_{t},
\end{aligned}
$$

where $b_{t}$ and $a_{t}$ are given by Eq. (17). Knowing $\mathcal{G}_{t}$ in Eq. (22), one obtains from Eq. (18) that

$$
\mathcal{U}_{t}^{\dagger}=e^{c_{t} b^{\dagger}\left(a^{\dagger}-a\right) b}=1+\left[e^{c_{t}\left(a^{\dagger}-a\right)}-1\right] b^{\dagger} b,
$$

where

$$
c_{t}=\left(g-g_{t}\right) E_{I} / E_{b}
$$

Therefore,

$$
\begin{gathered}
a_{t}=a\left(1-b_{t}^{\dagger} b_{t}\right)+b_{t}^{\dagger} a b_{t}, \\
b_{t}=e^{c_{t}\left(a^{\dagger}-a\right)} b .
\end{gathered}
$$

Analogous formulas hold for creation operators $a_{t}^{\dagger}$ and $b_{t}^{\dagger}$, obtained by Hermitian conjugation.

\section{A. Effective particle operators}

It is visible in Eq. (33) that the effective boson operators $a_{t}$ are equivalent to the bare ones in the subspace of Fock space without effective fermions, for in that case $b_{t} \equiv 0$.
In the subspace that contains one effective fermion, one has $b_{t}^{\dagger} b_{t} \equiv 1$ and is left with

$$
a_{t 1}=b_{t}^{\dagger} a b_{t}
$$

and a corresponding relation for $a_{t 1}^{\dagger}$. Evaluation yields

$$
a_{t 1}=\left(a+c_{t}\right) b^{\dagger} b
$$

and $a_{t 1}^{\dagger}$ is obtained by conjugation.

In summary, the annihilation operator for effective fermion, $b_{t}$, is given by Eq. (34), and the annihilation operator for an effective boson is

$$
a_{t}=a+c_{t} b^{\dagger} b
$$

where $c_{t}$ is given by Eq. (32). The corresponding creation operators are obtained by Hermitian conjugation. Using these results, one can check by a direct calculation that the effective Hamiltonian $H_{t}$ of Eq. (30) is equal to the initial Hamiltonian $H=H_{0}$ of Eq. (12).

\section{EXACT SPECTRUM IN THE FOCK SPACE}

One observes that there are three ways of seeking the model Hamiltonian spectrum. In the first way, one uses the Hamiltonian expressed in terms of the initial particle operators that correspond to $t=0$. In the second way, one uses the Hamiltonian expressed in terms of effective particle operators for some finite value of the RGPEP parameter $t$. The third way is reduced to inspection of the effective Hamiltonian with $t=\infty$. The respective forms of one and the same Hamiltonian $H=H_{0}$ of Eq. (12) are

$$
\begin{gathered}
H_{0}=E_{f} b^{\dagger} b+E_{b} a^{\dagger} a+g E_{I} b^{\dagger}\left(a^{\dagger}+a\right) b, \\
H_{t}=\left(E_{f}+g_{t}^{2} \Delta_{t}\right) b_{t}^{\dagger} b_{t}+E_{b} a_{t}^{\dagger} a_{t} \\
+g_{t} E_{I} b_{t}^{\dagger}\left(a_{t}^{\dagger}+a_{t}\right) b_{t}, \\
H_{\infty}=E_{\text {fermion }} b_{\infty}^{\dagger} b_{\infty}+E_{b} a_{\infty}^{\dagger} a_{\infty},
\end{gathered}
$$

where $\Delta_{t}$ is given in Eq. (28). Taking into account the commutation relations that the operators with $t=\infty$ obey, one sees that the eigenvalues are

$$
\begin{gathered}
E_{\text {fermion }}=\lim _{t \rightarrow \infty}\left(E_{f}+g_{t}^{2} \Delta_{t}\right) \\
=E_{f}-g^{2} E_{I}^{2} / E_{b}, \\
E_{n \text { bosons }}=n E_{b}, \\
E_{\text {fermion }+n \text { bosons }}=E_{\text {fermion }}+n E_{b},
\end{gathered}
$$

and the corresponding normalized eigenstates are 


$$
\begin{gathered}
\mid \text { fermion }\rangle=b_{\infty}^{\dagger}|0\rangle, \\
\mid n \text { bosons }\rangle=\frac{1}{\sqrt{n !}} a_{\infty}^{\dagger n}|0\rangle, \\
\mid \text { fermion }+n \text { bosons }\rangle=\frac{1}{\sqrt{n !}} a_{\infty}^{\dagger n} b_{\infty}^{\dagger}|0\rangle,
\end{gathered}
$$

where $|0\rangle$ denotes the model Hamiltonian ground state that contains no physical particles. According to Eqs. (33) and (34),

$$
\begin{gathered}
b_{\infty}=e^{g\left(E_{I} / E_{b}\right)\left(a^{\dagger}-a\right)} b, \\
a_{\infty}=a+g\left(E_{I} / E_{b}\right) b^{\dagger} b .
\end{gathered}
$$

A physical fermion state is composed of the bare fermion and a coherent state of bosons. Since $a^{\dagger}-a=$ $a_{t}^{\dagger}-a_{t}=a_{\infty}^{\dagger}-a_{\infty}$, one can speak of the coherent state of bare as well as effective or physical bosons. The $n$-boson eigenstates without a fermion are the same as if the interaction were absent.

\section{WEAK-COUPLING EXPANSION}

In the weak-coupling expansion, one hopes to gain some insight concerning solutions of a theory assuming that the coupling constant in the interaction terms is a very small number. After evaluating some quantity of interest using expansion in powers of an infinitesimal coupling, one can check how large the coupling would have to be for the result to match data. Then there comes the question of how large the remaining terms in the expansion are.

In the model with the coupling constant $g$ not very small, such procedure is not viable as an approximation method for obtaining eigenstates of the Hamiltonian $H$ in terms of bare particle operators that appear in its form $H_{0}$. This form corresponds to the Yukawa theory expressed in terms of bare degrees of freedom. Although the fermion eigenvalue $E_{f \infty}$ is just a quadratic function of $g$ and one might hope that an expansion up to terms order $g^{2}$ may be sufficient, the fermion eigenstate contains terms with all powers of the product $g$ times the bare boson creation operator acting on the vacuum state.

Quite different situation is encountered when one uses the Hamiltonian in its form $H_{t}$ with $E_{b}^{2} t$ sufficiently large for $g_{t}$ of Eq. (27) to be small. The eigenstates without a fermion are just free effective bosons created by $a_{t}^{\dagger}$ from the vacuum state. The eigenstates with a fermion are given by $b_{t}^{\dagger}|0\rangle$ plus admixtures of effective bosons that are created from the fermion state with strength $g_{t}$ instead of $g$. One sees in Eq. (27) that $g_{t}$ can be small for arbitrarily large $g$ when $t$ is made sufficiently large. In that case, the fermion state can be approximated well by using the expansion in powers of $g_{t}$.
The mechanism described above can be illustrated by the perturbative expansion up to second order for the fermion energy eigenvalue and the corresponding eigenstate. In general, a perturbative expansion is obtained by writing

$$
\begin{aligned}
|\psi\rangle= & \left(\psi_{00}+\psi_{01}+\psi_{02}+\cdots\right) b_{t}^{\dagger}|0\rangle \\
& +\left(\psi_{10}+\psi_{11}+\psi_{12}+\cdots\right) a_{t}^{\dagger} b_{t}^{\dagger}|0\rangle \\
& +\left(\psi_{20}+\psi_{21}+\psi_{22}+\cdots\right) a_{t}^{\dagger} a_{t}^{\dagger} b_{t}^{\dagger}|0\rangle+\cdots,
\end{aligned}
$$

where $\psi_{m n} \sim g_{t}^{n}$. The eigenvalue problem reads

$$
H_{t}|\psi\rangle=\left(E_{0}+E_{1}+E_{2}+\cdots\right)|\psi\rangle,
$$

where $E_{n}$ is of order $g_{t}^{n}$. Assuming that the dominant coefficient in front of $b_{t}^{\dagger}|0\rangle$ is $\psi_{00}$ of order 1 , one can limit the effective Fock-space expansion to only three terms: one effective fermion, one effective fermion and one effective boson, and one effective fermion and two effective bosons. Coefficients of the components with more effective particles are of order $g_{t}^{n}$ with $n>2$. By projecting both sides of Eq. (51) on these three basis states, one obtains three equations. Projection on the component $b_{t}^{\dagger}|0\rangle$ yields

$$
\begin{aligned}
0= & \left(E_{f}+g_{t}^{2} \Delta_{t}-E_{0}-E_{1}-E_{2}-\cdots\right) \\
& \times\left(\psi_{00}+\psi_{01}+\psi_{02}+\cdots\right) \\
& +g_{t} E_{I}\left(\psi_{10}+\psi_{11}+\psi_{12}+\cdots\right) .
\end{aligned}
$$

Projection on $a_{t}^{\dagger} b_{t}^{\dagger}|0\rangle$ leads to

$$
\begin{aligned}
0= & \left(E_{f}+g_{t}^{2} \Delta_{t}-E_{0}-E_{1}-E_{2}-\cdots\right) \\
& \times\left(\psi_{10}+\psi_{11}+\psi_{12}+\cdots\right) \\
& +E_{b}\left(\psi_{10}+\psi_{11}+\psi_{12}+\cdots\right) \\
& +g_{t} E_{I}\left(\psi_{00}+2 \psi_{20}+\psi_{01}+2 \psi_{21}+\cdots\right) .
\end{aligned}
$$

Projection on $a_{t}^{\dagger} a_{t}^{\dagger} b_{t}^{\dagger}|0\rangle$ produces

$$
\begin{aligned}
0= & 2\left(E_{f}+g_{t}^{2} \Delta_{t}-E_{0}-E_{1}-E_{2}-\cdots\right) \\
& \times\left(\psi_{20}+\psi_{21}+\psi_{22}+\cdots\right) \\
& +4 E_{b}\left(\psi_{20}+\psi_{21}+\psi_{22}+\cdots\right) \\
& +2 g_{t} E_{I}\left[\psi_{10}+\psi_{11}+\psi_{12}+\cdots\right] .
\end{aligned}
$$

Each of these equations contains terms proportional to powers of $g_{t}$. Equating coefficients of $1, g_{t}$ and $g_{t}^{2}$ on both sides of these equations, one arrives at a set of nine equations that must be satisfied simultaneously. Assuming that $\psi_{00}=1$, setting $\psi_{01}=\psi_{02}=0$ and introducing the normalization factor $N$, one obtains

$$
E=E_{f}-\frac{1}{E_{b}}\left(g_{t} e^{E_{b}^{2} t} E_{I}\right)^{2},
$$




$$
\begin{aligned}
|\psi\rangle= & N\left[b_{t}^{\dagger}|0\rangle-\frac{1}{E_{b}} g_{t} E_{I} a_{t}^{\dagger} b_{t}^{\dagger}|0\rangle\right. \\
& \left.+\frac{1}{2}\left(\frac{1}{E_{b}} g_{t} E_{I}\right)^{2} a_{t}^{\dagger} a_{t}^{\dagger} b_{t}^{\dagger}|0\rangle\right] .
\end{aligned}
$$

The term $\left(g_{t} E_{I}\right)^{2} / E_{b}$ in the effective fermion energy in Eq. (39) cancels the second-order self-interaction term that results from emission and absorption of an effective boson. Thus, even though in the model the fermion self-interaction is finite, the term $\left(g_{t} E_{I}\right)^{2} / E_{b}$ in the effective fermion energy term in the Hamiltonian $H_{t}$ appears in the role of a finite part of the fermion self-interaction counterterm when the parameter $t$ tends to zero and its inverse plays the role of a cutoff $\Lambda^{2}$. The finite part is positive, vanishes when $\Lambda \rightarrow 0$ or $t \rightarrow \infty$ and implies that in that limit the effective fermion energy in $H_{t}$ approaches the physical fermion eigenvalue $E_{\text {fermion. }}$. One could replace the effective fermion energy term in $H_{t}$ by the eigenvalue and ignore the selfinteraction effects.

\section{THE TAMM-DANCOFF APPROXIMATION}

The idea of the TD approximation [16,17] is to limit the Hamiltonian eigenvalue problem to a subspace of the Fock space defined by a limit on the number of virtual particles. One assumes that the eigenstate components with more particles than the limiting number have a small probability and can be neglected in the first approximation. Such approach was also proposed in the context of solving QCD in the front form of Hamiltonian dynamics, using the idea that a suitable renormalization group algorithm, including the Fock-sector-dependent counterterms, could be used to identify the dominant features of the dynamics as the limit on the number of particles is increased. Subsequently, one could attempt to compute corrections to the dominant picture using the methods of perturbative expansion and successive approximations [19], including some form of the coupling coherence [20].

In case of the RGPEP, the key feature that influences the accuracy of the TD type of approach to realistic theories is that instead of the bare, original field quanta one limits the number of the effective quanta. The idea is illustrated using Fig. 1. It shows plots of the expected number of virtual effective bosons in the physical fermion state as a function of the RGPEP scale parameter $t$. The plotted value is defined by

$$
\left\langle N_{t}\right\rangle=\left\langle\text { fermion }\left|a_{t}^{\dagger} a_{t}\right| \text { fermion }\right\rangle,
$$

where the fermion state is given in Eq. (45). Using Eqs. (37) and (48), one obtains

$$
\left\langle N_{t}\right\rangle=g_{t}^{2}\left(E_{I} / E_{b}\right)^{2}
$$

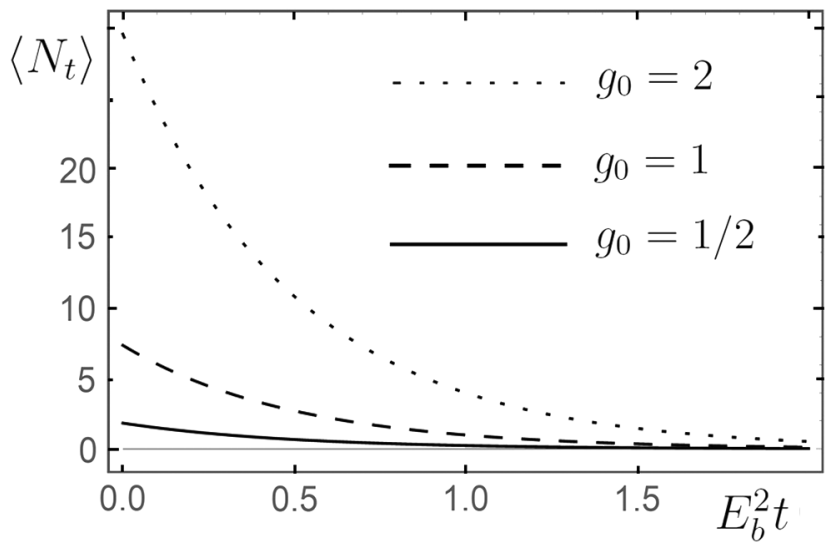

FIG. 1. Expectation value of the number of effective bosons, see Eq. (57), in the physical fermion eigenstate of Eq. (45) as a function of the RGPEP scale parameter $t$. The three curves correspond to the three values 2,1 , and $1 / 2$ of the coupling constant $g_{0}$ in Eq. (59), defined as the effective coupling constant $g_{t}$ for $t$ equal $t_{0}=1 / E_{b}^{2}$, assuming that the free boson energy $E_{b}$ equals the fermion-boson interaction energy parameter $E_{I}$ in the model Hamiltonian; see Sec. VII. It is visible that the TD approximation becomes increasingly accurate when $t$ increases, since $\left\langle N_{t}\right\rangle$ decreases exponentially fast with increase of $t$.

which for $E_{I}=E_{b}$ yields the expected number of virtual effective bosons in a physical fermion,

$$
\left\langle N_{t}\right\rangle=g_{t}^{2}=g_{0}^{2} e^{2 E_{b}^{2}\left(t_{0}-t\right)} .
$$

The coupling constant $g_{0}$ is the value that $g_{t}$ takes when $t=t_{0}$. We set the value of $t_{0}$ to $E_{b}^{-2}$, since this value of the running cutoff corresponds in magnitude to the energy change associated with emission or absorption of just one boson. The value of $g_{0}$ is arbitrary. To provide examples of the numbers involved, three values of the coupling constant $g_{0}$ are arbitrarily selected: 2,1 , and $1 / 2$. The three curves shown in Fig. 1 correspond to these three values of $g_{0}$. The number of virtual bosons in a physical fermion strongly depends on the value of $g_{0}$ and these three values are sufficient to illustrate the dependence. Each of the chosen values corresponds to a different value of the bare coupling constant $g$ in Eq. (12), $g=e g_{0}$.

It is visible in Fig. 1 that approximations of the TD type with just one or two virtual bosons do not apply in terms of the bare particles if the coupling constant $g_{0}$ is not sufficiently small. For example, if $g_{0}=2$, the expectation value $\left\langle N_{0}\right\rangle$ is almost 30. However, when $t$ grows, the expectation value $\left\langle N_{t}\right\rangle$ decreases. In the model, where one possesses the exact solution to the RGPEP equation, Fig. 1 shows that for $t$ exceeding $E_{b}^{-2}$ the effective interaction vertex suppression factor can become so small that the strength of the bare coupling constant is overcome and the TD approximation represents the physical fermion accurately in terms of a small number of the corresponding virtual effective particles. 


\section{TD HAMILTONIAN MATRICES}

If the coupling constant $g_{t}$ is sufficiently small and the parameter $t$ large enough for the RGPEP form factors to suppress the interaction terms in $H_{t}$ that change the number of effective particles, then the TD approximation may be valid. In that case, one can define the effective Hamiltonian matrices that describe the dynamics in terms of a limited number of effective Fock-space basis states. We call them the effective TD Hamiltonian matrices, or just TD matrices, denoted by $H_{\mathrm{TDt}}$. One can compute them following the pattern illustrated below in terms of our model.

Consider the Hamiltonian $H_{t}$ in which the effective, particle number-changing interaction term is weak enough to expect that the TD approximation is reasonable. Suppose one is interested in an approximate computation of observables for a physical fermion. In the model, the physical fermion is known exactly. It is represented by the state $\mid$ fermion $\rangle$ in Eq. (45). However, in an approximate calculation in a realistic theory, a physical fermion state would not be known exactly.

Suppose one expects that the physical fermion state is dominated by the basis state $b_{t}^{\dagger}|0\rangle$, while the basis state $a_{t}^{\dagger} b_{t}^{\dagger}|0\rangle$ provides the leading correction. Still smaller corrections involve the basis states $a_{t}^{\dagger n} b_{t}^{\dagger}|0\rangle$ with $n>1$. To describe the physical fermion state using the TD approximation, one computes the matrix $H_{\mathrm{TD}}$ that acts on the coordinates of states in the subspace of the Fock space that is spanned by the basis states with one effective fermion and a limited number of effective bosons. If instead of the physical fermion one were interested in the properties of states $\mid$ fermion $+n_{b}$ bosons $\rangle$, one would first compute Hamiltonian matrix $H_{\mathrm{TD}}$ that acts on the coordinates in the subspace spanned by the effective basis states $a_{t}^{\dagger n_{b}-1} b_{t}^{\dagger}|0\rangle$, $a_{t}^{\dagger n_{b}} b_{t}^{\dagger}|0\rangle$, and $a_{t}^{\dagger n_{b}+1} b_{t}^{\dagger}|0\rangle$. Corrections would follow from enlarging the matrix to include coordinates in directions of basis states with $n_{b} \pm 2$ effective bosons, etc.

A simple illustration of the TD approximation is obtained in case of the physical fermion and the assumption that the matrix $H_{\mathrm{TDt}}$ only acts on the three-dimensional vectors of coordinates in the Fock subspace spanned by the basis states $b_{t}^{\dagger}|0\rangle, a_{t}^{\dagger} b_{t}^{\dagger}|0\rangle$ and $a_{t}^{\dagger 2} b_{t}^{\dagger}|0\rangle / \sqrt{2}$. The first approximation is obtained by writing

$\mid$ Fermion $\left._{\mathrm{TD}}\right\rangle=x_{t 0} b_{t}^{\dagger}|0\rangle+x_{t 1} a_{t}^{\dagger} b_{t}^{\dagger}|0\rangle+\frac{1}{\sqrt{2}} x_{t 2} a_{t}^{\dagger 2} b_{t}^{\dagger}|0\rangle$.

Then one observes that the physical fermion eigenvalue problem has the form

$$
\begin{gathered}
H_{t}|\psi\rangle=E_{\mathrm{TD}}|\psi\rangle, \\
\left.|\psi\rangle=\mid \text { Fermion }_{\mathrm{TD}}\right\rangle+\left|n_{b}>2\right\rangle,
\end{gathered}
$$

where $\left|n_{b}>2\right\rangle$ stands for all components with more effective bosons than 2. Projecting this equation on the same range of components that appears in Eq. (60), one obtains the matrix equation

$$
\begin{aligned}
& {\left[\begin{array}{ccc}
h_{2,2} & h_{2,1} & 0 \\
h_{1,2} & h_{1,1} & h_{1,0} \\
0 & h_{0,1} & h_{0,0}
\end{array}\right]\left[\begin{array}{l}
x_{t 2} \\
x_{t 1} \\
x_{t 0}
\end{array}\right]+\left[\begin{array}{c}
h_{2, n_{b}>2} \\
0 \\
0
\end{array}\right]} \\
& =E_{\mathrm{TD}}\left[\begin{array}{l}
x_{t 2} \\
x_{t 1} \\
x_{t 0}
\end{array}\right],
\end{aligned}
$$

where the matrix elements are

$$
h_{m, n}=\frac{1}{\sqrt{m ! n !}}\left\langle 0\left|b_{t} a_{t}^{m} H_{t} a_{t}^{\dagger n} b_{t}^{\dagger}\right| 0\right\rangle,
$$

with $0 \leq m, n \leq 2$. The TD approximation amounts to setting $h_{2, n_{b}>2}=0$. The effective TD Hamiltonian matrix is defined by

$$
H_{\mathrm{TDtmn}}=h_{m, n} .
$$

Its eigenvalue problem reads

$$
\sum_{n=0}^{2} H_{\mathrm{TDtmn}} x_{n}=E_{\mathrm{TD}} x_{m} .
$$

One has

$$
\begin{gathered}
H_{\mathrm{TD} t 22}=E_{f}+g_{t}^{2} \Delta_{t}+2 E_{b}, \\
H_{\mathrm{TD} \mathrm{t} 21}=H_{\mathrm{TD} 12 \mathrm{t}}=\sqrt{2} g_{t} E_{I}, \\
H_{\mathrm{TDt} 11}=E_{f}+g_{t}^{2} \Delta_{t}+E_{b}, \\
H_{\mathrm{TD} t 10}=H_{\mathrm{TD} 01 \mathrm{t}}=g_{t} E_{I}, \\
H_{\mathrm{TD} \mathrm{t} 00}=E_{f}+g_{t}^{2} \Delta_{t} .
\end{gathered}
$$

The eigenvalues $E$ written in the form $E=E_{f}+g_{t}^{2} \Delta_{t}+$ $x E_{b}$ obey the equation

$$
(2-x)(1-x) x+\alpha(2-3 x)=0
$$

where $\alpha=\left(g_{t} E_{I} / E_{b}\right)^{2}$. If $\alpha$ were zero due to $g_{t}=0$, the three eigenvalues $E_{n}=E_{f}+g_{t}^{2} \Delta_{t}+x_{n} E_{b}$ with $x_{n}=n$ would correspond to a free effective fermion and $n$ free bosons. Assuming that $x=n+y \alpha$ and neglecting higher powers of $\alpha$, one obtains $E_{n}=E_{\text {fermion }}+n E_{b}$ for $n$ equal 0 or 1 , as expected on the basis of the exact solution given in Eq. (44), and Eq. (45) or (47), respectively. Higher order terms in the expansion of $x$ in powers of $\alpha$ can be used to 
compare the TD approximation with the weak-coupling expansion. Next level of the TD approximation would be obtained by introducing the component $a_{t}^{\dagger 3} b_{t}^{\dagger}|0\rangle / \sqrt{6}$ and neglecting $h_{2, n_{b}>3}$.

The coordinates $x_{n}$ in realistic theories would not be just numbers but unknown functions of only $3 n$ relative momentum variables and discrete quantum numbers of fermions and bosons. The number of momentum arguments would be the same in the nonrelativistic and relativistic theories because the total momentum of the eigenstates drops out from the TD matrix problem and the eigenvalues $E$ are solely the masses squared of the physical systems.

One can use Eq. (63), ignoring $h_{2, n_{b}>2}$, to evaluate the Fock-space coordinate $x_{t 2}$ in terms of the coordinates $x_{t 1}$ and $x_{t 0}$ using a fully nonperturbative Gaussian elimination or the so-called $R$ operation, the latter when either the boson energy $E_{b}$ is large [21] or $g_{t}$ is small. The Gaussian elimination yields

$$
x_{t 2}=\frac{1}{E_{\mathrm{TD}}-h_{2,2}} h_{2,1} x_{t 1},
$$

which can be put into the remaining two equations. The result is

$$
\begin{gathered}
{\left[\begin{array}{cc}
h_{1,1}+h_{1,2} \frac{1}{E_{\mathrm{TD}}-h_{2,2}} h_{2,1} & h_{1,0} \\
h_{0,1} & h_{0,0}
\end{array}\right]\left[\begin{array}{l}
x_{t 1} \\
x_{t 0}
\end{array}\right]} \\
=E_{\mathrm{TD}}\left[\begin{array}{l}
x_{t 1} \\
x_{t 0}
\end{array}\right] .
\end{gathered}
$$

This is the TD matrix eigenvalue problem including the fermion self-interaction in the fermion-boson component. Note that the eigenvalue $E_{\mathrm{TD}}$ appears on both sides of the problem, which requires a nonperturbative matching of its left-hand side value with its value on the right-hand side. If instead of the Gaussian elimination one used the operation $R$ [21] in expansion up to second power of the coupling constant $g_{t}$, including the perturbative orthogonality and normalization corrections, then the eigenvalue $E_{\mathrm{TD}}$ in Eq. (74) on the left-hand side would be replaced by $E_{f}+E_{b}$. Even though in this case the left-hand side matrix would only contain terms of order up to $g_{t}^{2}$, the eigenstates would depend on $g_{t}$ in a way specific to the particular TD approximation. The issue would then be what changes occur when one attempts to improve the approximation by including more effective particles or higher powers of $g_{t}$ in the TD Hamiltonian matrices. An example of a phenomenological study based on the hypothesis that gauge bosons obtain an effective mass is presented in [22] in the case of description of baryons using heavy-flavor QCD.

Examples of perturbative and TD approximations described above and in Secs. VI and VII in the exactly solvable model can be used in assessing convergence of similar approximations in more complex cases. Consider the numerical studies of eigenvalue problems for TD Hamiltonian matrices obtained using bare quanta in the Yukawa and Yukawa-like theories, such as reported in $[23,24]$ and references therein. The same theories can be considered in the limit of fermion and boson masses much larger than the cutoff parameters, irrespective of the form of regularization. One can limit numerical calculations, where the quantum degrees of freedom are discrete, to a single mode for all quanta involved, precisely as it is done here in the Yukawa theory to obtain our model Hamiltonian. In that setup, the TD Hamiltonian matrices one would obtain would resemble the ones in our model. One can compare the accuracy and convergence measures adopted in [23,24] with exact results shown in Fig. 1.

In our model case, the bare coupling constant $g=e g_{0}$, see Eq. (27), determines the expectation value for the number of bare bosons, $\left\langle N_{0}\right\rangle$, in the exact fermion eigenstate. For $g$ small, a small $\left\langle N_{0}\right\rangle$ is expected. However, for $g$ order $\sqrt{4 \pi} \sim 3.5$, which corresponds to the conventional coupling constant $g^{2} /(4 \pi) \sim 1$, Fig. 1 shows that the expected number of bare bosons exceeds 30 . It is stated in $[23,24]$ that in theories considered there one achieves convergence using TD matrices with three or four bosons for quite large coupling constants. It would hence be of interest to find out what mechanism is at work by which the inclusion of additional interactions and motion of bare bosons with respect to bare fermions improves the convergence so significantly. Convergence for the electromagnetic form factors may be less indicative of the number of bosons needed because the contributions of the Fock components with $n$ constituents at large momentum transfers may quickly decrease with $n$ [25].

The fact that the TD matrix eigenvalue problems are particularly suitable as a tool for seeking approximate solutions to QFT in the FF of Hamiltonian dynamics originates in the special circumstance that the momentum component $p^{+}$is conserved by the interactions and cannot be negative, in a sharp distinction from the momentum component $p^{z}$ in the IF of dynamics, which can have both signs. As a result, discretization of momenta in a box on a front divides the available total momentum $P^{+}$of an eigenstate of a FF Hamiltonian into a definite number of pieces, say $K$. Each Fock-space constituent of an eigenstate must carry a natural number of units $P^{+} / K$. Therefore, the number of constituents is limited from above by $K$, which ties the maximal number of constituents in the TD approximation to the resolution of momentum discretization, $K$. This is the basis of the so-called discretized lightcone quantization (DLCQ) [26-28].

The DLCQ method has been applied to the Yukawa theory [29]. Divergences were regulated using the PauliVillars method that introduces additional massive fields. To obtain solvable models, the masses of quanta of the 
additional fields were set equal to those of the corresponding physical ones [30]. Similar DLCQ computations were also carried out in a solvable model that closely resembles the Yukawa theory of heavy fermions [31,32]. That model was used in [31] to introduce the concept of "clothed" particles. The clothed particle was defined using an exact solution for a state of a single particle. An analogous solution was recovered using DLCQ. In these examples, the DLCQ methods were found useful for constructing lowmass states in which the mean number of bare constituents was small.

As resulting from simplifications of one and the same Yukawa theory, the applications of the DLCQ mentioned above allow one to pin point basic features by which the RGPEP and DLCQ approaches differ. These features are visible in Eqs. (38)-(40) of Sec. V. They display three distinct operator forms of the same Hamiltonian that acts in the model Fock space.

Equation (38) corresponds to the initial, one might say, canonical Hamiltonian of a theory without counterterms. This operator provides the starting point for the RGPEP, which is set up at the scale parameter $t=0$. Since the model is ultraviolet finite, no ultraviolet divergences need to be countered.

Equation (39) displays the same Hamiltonian written in terms of creation and annihilation operators for the effective particles that correspond to an arbitrary positive value of the finite scale parameter $t$, as described in Sec. IV. The formula displays an effective fermion-boson interaction term and a fermion self-interaction term. The Hamiltonian has the universal form of a polynomial function of effective particle operators. The polynomial coefficients and operators are the computed functions of $t$. The self-interaction term vanishes at $t=0$ because there are no counterterms needed in the initial Hamiltonian. If instead the initial Hamiltonian led to divergences in any term of $H_{t}$ for any finite $t$, one would compute the counterterms at $t=0$ by demanding that the divergences in $H_{t}$ are eliminated. The model is too simple to illustrate in detail what is done in the RGPEP regarding computation of counterterms when the initial Hamiltonian is divergent. However, detailed perturbative illustrations are available in an asymptotically free example of a scalar theory in $5+1$ dimensions [33] and in a general derivation of formulas for relativistic Hamiltonians of effective particles in QFT [34]. Here it is only noted that in the presence of divergent selfinteractions, the self-interaction term would include a free, finite part of the corresponding counterterm. That part would be adjusted by comparison with experiment and may be constrained by demands of symmetry that the resulting theory is meant to possess.

Equation (40) is an expression of the same model Hamiltonian in terms of the operators that create physical states from the vacuum state. A state of a single physical particle is an eigenstate of the Hamiltonian.
The formula (40) is obtained in the limit $t \rightarrow \infty$. The effective creation and annihilation operators labeled by $\infty$ correspond to the physical particles of the model. Generally, $H_{\infty}$ that comes out of solving the RGPEP equation could involve mixing of eigenstates within degenerate multiplets that in addition to the Hamiltonian eigenvalues are labeled by the eigenvalues of other operators that commute with $H_{t}$, such as a component of the angular momentum, spin, isospin, or a similar quantity. Our model Hamiltonian form of Eq. (40) corresponds to both the concept of clothed particles in [31] and the DLCQ solutions for single physical particle states. It is visible in Eq. (40) that the model of Eq. (12) is too simple to produce interactions between the effective particles that correspond to $t=\infty$ and match physical ones as single-particle states. It is worth stressing that the effective particles for $t \rightarrow \infty$ do not have to correspond to the physical ones. This is important for considerations that involve the concept of confinement; see below and Sec. X.

It is now clear that the RGPEP and DLCQ computations discussed above differ significantly. The RGPEP produces a whole family of equivalent effective Hamiltonians. The DLCQ does not produce such a family. It does label Hamiltonian matrices with the resolution $K$ and the transverse momentum cutoff, introduced by the Pauli-Villars masses. However, these are the regularization parameters. The resolution $K$ and the Pauli-Villars masses are meant to be sent to infinity in order to obtain solutions of a theory. They are not the finite parameters analogous to the RGPEP $t$ on which the physical quantities do not depend; see Sec. V. Each member of the family labeled by $t$ is expressed using a different choice of degrees of freedom in one and the same theory, which means using different creation and annihilation operators, or different quantum field operators that are built from them. Solving the TD Hamiltonian matrix eigenvalue problems in terms of bare quanta for which $t=0$ may be very difficult numerically because of involvement of many basis states in the dynamics, as is illustrated in the model by Fig. 1. An effective Hamiltonian with a finite $t$ that is adjusted to the scale of the physical quantity of interest is dominated by the effective basis states of a similar scale. An approximate but accurate description of the quantity of interest is simpler to achieve that way than by keeping all bare basis states in a computation that requires handling of all variables of the theory up to the cutoffs. The model example illustrates this feature solely in terms of the magnitude of the effective coupling constant that decreases as $t$ increases and thus weakens the coupling between different effective Fock components that correspond to the parameter $t$. In contrast, the DLCQ approach attempts to solve the theory directly in terms of the degrees of freedom present in the quantum Hamiltonian in its initial form, analogous to Eq. (38), i.e., the one that is obtained by quantization of a local theory. 
Our model example makes it also clear that the concept of clothed particles mentioned above differs from the RGPEP concept of scale-dependent effective particles. The clothed particles approach is based on writing a Hamiltonian in terms of operators associated with the physical particles instead of the bare ones. In the RGPEP language, the idea is to replace the gradual evolution from $t=0$ to $t=\infty$ by a single jump. Such replacement is not available in any closed form in complex theories for which one does not have any exact solutions. Notably, in case of confinement, the required physical particles are not supposed to exist. The issue is relevant to the ultimate DLCQ limit $K \rightarrow \infty$ that appears to be related to questions concerning the vacuum, which is assumed to carry $p^{+}=0$. The RGPEP approach is conceptually different from the clothed particle approach. Its equations can be solved for effective operators making various guesses or approximations and the resulting effective particles do not have to be identified with any physical, individually observable objects. The effective Hamiltonians $H_{t}$ can be studied in terms of their predictions for quantities accessible experimentally. For an example of such attempt in heavy-flavor QCD, see [22].

Another basic feature that distinguishes the RGPEP example from the DLCQ examples mentioned above is that the number of quantum degrees of freedom stays the same in the effective theory for all values of $t$, including the canonical theory at $t=0$. However, the interaction terms in $H_{t}$ evolve with $t$ as the RGPEP Eq. (15) dictates. If the initial theory were divergent, the ultraviolet counterterms would be computed in the process of solving Eq. (15) and they would be inserted in the initial condition at $t=0$. They would thus not be constructed by adding degrees of freedom like in the Pauli-Villars approach. Instead, the demand on the RGPEP evolution that for finite $t$ it yields finite effective Hamiltonians $H_{t}$ would be used to determine the missing counterterms in $H_{0}$.

Finally, it should be observed that in the nonrelativistic contexts of condensed matter physics, addressed broadly in [35], as well as in nuclear physics theory developed in [36] and elsewhere, similar Wegner-like equations and corresponding TD Hamiltonian matrix eigenvalue problems appear that resemble the ones obtained by applying the RGPEP to the model Hamiltonian of Eq. (12) or other model Hamiltonians of analogous nature, cf. [37]. According to the rule that the same equations have the same solutions, no matter what their interpretation is, and in view of the discussion of this section, it becomes clear that the RGPEP concept of effective particles developed in particle physics and explicitly illustrated using the elementary Eq. (39), can be introduced in the other branches of physical theory as well. For example, one can attempt to introduce a whole family of scale-dependent effective electron operators that include phonon operators in a model of a condensed-matter medium or effective nucleon operators that include meson operators in a model of a nucleus.

\section{MODEL SOLUTION AND REALISTIC THEORIES}

The model solution illustrates the structure, function, and purpose of the RGPEP in the context where no divergences appear. The concept of counterterms only shows up through the cancellation of the fermion self-interaction energy, due to emission and absorption of bosons, against the effective fermion energy in the eigenvalue problem for the Hamiltonian $H_{t}$. The terms that cancel out are finite. The pattern is analogous to the cancellation between the finite parts of counterterms and self-interactions in realistic theories.

The model solution illustrates the weakening of effective interactions solely in terms of the coupling constant that decreases as the RGPEP evolution parameter $t$ grows. This weakening corresponds to the weakening obtained in terms of the vertex form factors in realistic theories. The model running-coupling constant corresponds to the vertex form factor for the specific value of its argument, corresponding to the invariant mass change caused by the interaction. Emergence of the RGPEP vertex form factors in the Yukawa theory is described in [38]. Analogous appearance of the vertex form factors in the Abelian gauge theory is shown in [15]. The RGPEP form factors that emerge in the third-order computation of the effective vertices in a nonAbelian theory is provided in [39].

Extension of the model solution that would include the motion of bosons with respect to fermions and hence produce the associated vertex form factors, would be of great value. As pointed out earlier, the RGPEP vertex form factors are expected to be important in the derivation of effective quark and gluon dynamics in QCD. However, given the complexity of QCD, one might attempt to first undo some of the model simplifications made here and tackle the problem of applying the RGPEP to the Yukawa theory. To be specific, one may aim at a comprehensive resolution of the paradox that concerns interactions of nucleons with pions, and perhaps also other mesons. Namely, the exchange of just one pion between nucleons yields the Yukawa potential in second-order perturbation theory, but the coupling constant one needs to introduce in order to match the phenomenology is so large that the standard perturbation theory with local interactions cannot be valid. Perhaps the large coupling corresponds not to a canonical Yukawa theory with $t=0$ but to the effective theory in which $t$ corresponds to the pion mass scale and the vertex form factors make the interaction effectively quite weak by suppressing it outside the small momentum transfer range that corresponds to the pion exchange.

The model solution includes a coherent state of bosons around a fermion. One could ask if the pattern exhibited by the model could be followed for the purpose of explaining 
if the effective Yukawa theory could describe the pion cloud around nucleons.

Since the RGPEP suppression of interactions corresponds to the vertex form factors, it makes sense to ask if any theory that introduces vertex form factors of some width might correspond to an effective one in the sense of the RGPEP for a width parameter $t$ matching the form factor scale. The example of particular interest is provided by the Nambu and Jona-Lasinio model [40] that to the author's best knowledge was never analyzed using the RGPEP.

\section{CONCLUSION}

The main import of the elementary model study is that it illustrates how the RGPEP works in an exactly solvable model. However, the realistic theories are much more complex than the model and one cannot predict on the model basis if the RGPEP can fully provide the means that are required for unambiguous identification of the corresponding effective Hamiltonians in complex theories. To find out what can be achieved in that matter, one would have to focus on the direct application of the RGPEP in terms of perturbative expansions and TD approximations to the complex theories. In that context, the model solution is of value because such approximate methods quickly get quite convoluted in realistic theories. The value is that one can use the model as a pattern to follow and to consult with when calculations get hard to see through. The key example of a barrier to break is to solve the RGPEP equation up to the fourth order of perturbation theory in QCD and derive the corresponding TD Hamiltonian matrices. Perhaps this is the way to obtain the constituent-quark picture of hadrons from QCD.

The case of quarks in QCD is particularly pressing even though one can also try to use the RGPEP for addressing theoretical issues of the Standard Model as a whole. The idea of constructing effective quarks dates back to early years of current algebra [41]. As far as the author knows, it is not fully realized till today, while the particle data tables [42] continue to classify hadrons mostly in terms of just two or three quark constituents. States that contain two more quarks are being added in the same spirit of constituents. QCD suggests instead that hadrons are built from practically unlimited numbers of quarks, antiquarks, and gluons of canonical theory. Despite the great progress of lattice gauge theory, Gell-Mann's opinion from twenty years ago [43] appears still valid: "The mathematical consequences of QCD have still not been properly extracted, and so, although most of us are persuaded that it is the correct theory of hadronic phenomena, a really convincing proof still requires more work. It may be that it would be helpful to have some more satisfactory method of truncating the theory, say by means of collective coordinates, than is provided by the brute-force lattice gauge theory approximation!"
The author's opinion is that the basic difficulty to overcome before one can address precise phenomenology that involves fast moving and strongly interacting hadrons, is to first somehow gain control of the ground state of the theory. The reason is that all particle states one considers are meant to be created by action of operators on that special state. Such control is also desired concerning spontaneous breaking of symmetries. In the FF of Hamiltonian dynamics, the vacuum problem is formulated in a different way than in the IF dynamics; e.g., see [44]. The condition $p^{+}>0$ for all quanta with finite momenta and nonzero masses can be compared with the condition that the vacuum state carries zero momentum. The vacuum state should also be invariant with respect to a change of an inertial frame of reference. This may be a large change, such as to the infinite moment frame used in the parton model. Somehow the vacuum state is limited to states akin to those with $p^{+}=0$, sometimes called the FF zero modes.

The vacuum problem of QCD has a long history, stimulated by the concepts of quark and gluon condensates and posing questions in cosmology. To gain a perspective, one can consult the works [45-47]. The leading condensates can be simply incorporated in the FF version of QCD sum rules [48] using the condition $p^{+}>\epsilon^{+}$for all nonvacuum modes while the vacuum modes must have $p^{+}<\epsilon^{+}$. The constant $\epsilon^{+}$is treated as infinitesimal. If one assumed that the states with momenta $p^{+}<\epsilon^{+}$were absent, one could even think that the cosmological vacuum problem may be resolved [49]. However, the dynamics of modes with $p^{+}<\epsilon^{+}$is singular and to the author's best knowledge it is not understood.

Of course, the exact computation of effective Hamiltonians for the model of Eq. (12) is only relevant to the vacuum issue because the computation is used to illustrate the RGPEP. The point is that the vacuum problem in the FF Hamiltonians can be turned into a renormalization group issue according to [2]. Namely, the counterterms to the cutoff $\epsilon^{+} \rightarrow 0$ are expected to mimic vacuum effects and one hopes to finesse dynamical effects due to the latter that way. The idea is presented in [2] using the FF power counting and original similarity renormalization group procedure [12,13]. However, the number and complexity of terms one obtains turns out difficult to handle using the similarity procedure. With the RGPEP, the situation is different because one does not need to directly address the multitude of matrix elements of many complex operators that involve initially unknown functions of many momentum variables. Therefore, one can focus instead on behavior of coefficients in polynomial functions of creation and annihilation operators for effective particles. Moreover, the RGPEP equation in QCD that corresponds to Eq. (15) in our model discussion secures invariance of the Hamiltonians $H_{t}$ with respect to seven kinematical Poincaré symmetries, leaving only three that are dynamical 
and need to be renormalized. Consequently, instead of the cutoff $p^{+}>\epsilon^{+}$on the absolute momenta $p^{+}$, one can use a dimensionless cutoff $x>\epsilon$ on the ratio $x=p_{1}^{+} / p_{2}^{+}$that momenta of particles 1 and 2 involved in an interaction term can form.

Exact nonperturbative solutions of the RGPEP equation in QFT as complex as QCD are not currently foreseeable. However, one can study the terms that emerge in perturbative expansion using asymptotic freedom, known in the FF effective particle Hamiltonians to the lowest order only [39]. The fourth-order calculation mentioned earlier is of interest because this is the first place where the running coupling appears in the effective interaction terms and increases with $t$. General fourth-order RGPEP formulas are available in [34]. As long as the effective coupling constant is not too large, one can use the perturbative expansion to learn what kinds of terms arise. Initial attempts at phenomenology included only second-order formulas for $H_{t}$ in QCD of heavy quarks [22], assuming that gluons gain effective masses. One needs to understand what happens in fourth order to see if there are any signs of development of constituent quark masses for light quarks. If the masses emerge and the coupling constant stays small enough, one can take advantage of the exact model and follow its pattern toward systematic improvement in accuracy of the computation.
[1] K. G. Wilson, Model hamiltonians for local quantum field theory, Phys. Rev. 140, B445 (1965).

[2] K. G. Wilson, T. S. Walhout, A. Harindranath, W.-M. Zhang, R. J. Perry, and S. D. Głazek, Nonperturbative QCD: A weak-coupling treatment on the light front, Phys. Rev. D 49, 6720 (1994); Fig. 6.

[3] E. M. Henley and W. Thirring, Elementary Quantum Field Theory (McGraw-Hill, New York, 1962).

[4] T. D. Lee, Some special examples in renormalizable field theory, Phys. Rev. 95, 1329 (1954).

[5] H. J. Lipkin, N. Meshkov, and A. J. Glick, Validity of manybody approximation methods for a solvable model: (I). Exact solutions and perturbation theory, Nucl. Phys. 62, 188 (1965).

[6] N. Debergh and Fl. Stancu, On the exact solutions of the Lipkin-Meshkov-Glick model, J. Phys. A 34, 3265 (2001).

[7] C. Providência, J. da Providência, Y. Tsue, and M. Yamamura, The Lipkin Model in Many-Fermion System as an Example of the su(1,1) $\otimes \mathrm{su}(1,1)$-Algebraic Model, Prog. Theor. Phys. 116, 87 (2006).

[8] J. C. Louw, J. N. Kriel, and M. Kastner, Thermalization of a Lipkin-Meshkov-Glick model coupled to a bosonic bath, Phys. Rev. A 100, 022115 (2019).

[9] P. A. M. Dirac, Forms of relativistic dynamics, Rev. Mod. Phys. 21, 392 (1949).

[10] S. D. Głazek and R. J. Perry, Fixed sources in light-front dynamics and Wilson's model of coupling-constant renormalization, Phys. Rev. D 45, 3734 (1992).

[11] S. D. Głazek and T. Masłowski, Renormalized Poincaré algebra for effective particles in quantum field theory, Phys. Rev. D 65, 065011 (2002).

[12] S. D. Głazek and K. G. Wilson, Renormalization of Hamiltonians, Phys. Rev. D 48, 5863 (1993).

[13] S. D. Głazek and K. G. Wilson, Perturbative renormalization group for Hamiltonians, Phys. Rev. D 49, 4214 (1994).

[14] F. Wegner, Flow-equations for Hamiltonians, Ann. Phys. (N.Y.) 506, 77 (1994).
[15] S. D. Głazek, Computation of effective front form Hamiltonians for massive Abelian gauge theory, Phys. Rev. D 101, 034005 (2020).

[16] Ig. Tamm, Relativistic interaction of elementary particles, J. Phys. USSR 9, 449 (1945).

[17] S. M. Dancoff, Non-adiabatic meson theory of nuclear forces, Phys. Rev. 78, 382 (1950).

[18] S. Szpigel and R. J. Perry, in Quantum Field Theory, A 20th Century Profile, edited by A. N. Mitra (National Physical Lab, New Delhi, India, 2000), p. 59, https://arxiv.org/pdf/ hep-ph/0009071.pdf.

[19] R. J. Perry, A. Harindranath, and K. G. Wilson, Light-front Tamm-Dancoff field theory, Phys. Rev. Lett. 65, 2959 (1990).

[20] R. J. Perry and K. G. Wilson, Perturbative renormalizability with an infinite number of relevant and marginal operators, Nucl. Phys. B403, 587 (1993).

[21] K. G. Wilson, Model of Coupling-Constant Renormalization, Phys. Rev. D 2, 1438 (1970).

[22] K. Serafin, M. Gómez-Rocha, J. More, and S. D. Głazek, Approximate Hamiltonian for baryons in heavy-flavor QCD, Eur. Phys. J. C 78, 964 (2018).

[23] Y. Li, V. A. Karmanov, P. Maris, and J. P. Vary, Ab initio approach to the non-perturbative scalar Yukawa model, Phys. Lett. B 748, 278 (2015).

[24] V. A. Karmanov, Y. Li, A. V. Simonov, and J. P. Vary, Nonperturbative solution of scalar Yukawa model in twoand three-body Fock space truncations, Phys. Rev. D 94, 096008 (2016).

[25] S. J. Brodsky and G. R. Farrar, Scaling laws for largemomentum-transfer processes, Phys. Rev. D 11, 1309 (1975).

[26] H. C. Pauli and S. J. Brodsky, Solving field theory in one space and one time dimension, Phys. Rev. D 32, 1993 (1985).

[27] H. C. Pauli and S. J. Brodsky, Discretized light-cone quantization: Solution to a field theory in one space and one time dimension, Phys. Rev. D 32, 2001 (1985). 
[28] S. J. Brodsky, H-C. Pauli, and S. S. Pinsky, Quantum chromodynamics and other field theories on the light cone, Phys. Rep. 301, 299 (1998).

[29] S. J. Brodsky, J. R. Hiller, and G. McCartor, Pauli-Villars regulator as a nonperturbative ultraviolet regularization scheme in discretized light-cone quantization, Phys. Rev. D 58, 025005 (1998).

[30] S. J. Brodsky, J. R. Hiller, and G. McCartor, Exact solutions to Pauli-Villars-regulated field theories, Ann. Phys. 296, 406 (2002).

[31] O. W. Greenberg and S.S. Schweber, Clothed particle operators in simple models of quantum field theory, Nuovo Cimento 8, 378 (1958).

[32] S. S. Schweber, An Introduction to Relativistic Quantum Field Theory (Row, Peterson and Co., Evanston, IL, 1961), p. 339.

[33] S. D. Głazek, Boost-invariant running couplings in effective Hamiltonians, Phys. Rev. D 60, 105030 (1999).

[34] S. D. Głazek, Perturbative Formulae for Relativistic Interactions of Effective Particles, Acta Phys. Pol. B 43, 1843 (2012).

[35] S. Kehrein, The Flow Equation Approach to Many-Particle Systems, Springer Tracts in Modern Physics Vol. 217 (2006), https:/www.springer.com/gp/book/9783540340676.

[36] S. K. Bogner, R. J. Furnstahl, and R. J. Perry, Similarity renormalization group for nucleon-nucleon interactions, Phys. Rev. C 75, 061001 (2007).

[37] G. Ciobanu, V. Bârsan, and A. T. Mincu, Hamiltonian renormalization with applications to the quantum quartic oscillator, Rom. J. Phys. 55, 539 (2010), http://www.nipne .ro/rjp/2010_55_5-6/0539_0568.pdf.
[38] S. D. Głazek and M. Więckowski, Large-momentum convergence of Hamiltonian bound-state dynamics of effective fermions in quantum field theory, Phys. Rev. D 66, 016001 (2002).

[39] M. Gómez-Rocha and S. D. Głazek, Asymptotic freedom in the front-form Hamiltonian for quantum chromodynamics of gluons, Phys. Rev. D 92, 065005 (2015).

[40] Y. Nambu and G. Jona-Lasinio, Dynamical model of elementary particles based on an analogy with superconductivity. II, Phys. Rev. 124, 246 (1961).

[41] H. J. Melosh, Quarks: Currents and constituents, Phys. Rev. D 9, 1095 (1974).

[42] P. A. Zyla et al. (Particle Data Group), Review of particle physics, Prog. Theor. Exp. Phys. (2020), $083 \mathrm{C} 01$.

[43] M. Gell-Mann, Quarks, Color, and QCD, in The Rise of the Standard Model, edited by L. Hoddeson et al. (Cambridge University Press, Cambridge, United Kingdom, 1999), p. 633.

[44] J. B. Kogut and L. Susskind, The parton picture of elementary particles, Phys. Rep. 8, 75 (1973).

[45] M. A. Shifman, A. I. Vainshtein, and V. A. Zakharov, QCD and resonance physics. theoretical foundations, Nucl. Phys. B147, 385 (1979).

[46] S. Weinberg, The cosmological constant problem, Rev. Mod. Phys. 61, 1 (1989).

[47] S. Weinberg, Ultraviolet divergences in cosmological correlations, Phys. Rev. D 83, 063508 (2011).

[48] S. D. Głazek, Light-front QCD in the vacuum background, Phys. Rev. D 38, 3277 (1988).

[49] S. J. Brodsky and R. Shrock, Condensates in quantum chromodynamics and the cosmological constant, Proc. Natl. Acad. Sci. U.S.A. 108, 45 (2011). 